Published in final edited form as:

AIDS. 2012 July 17; 26(11): 1345-1354. doi:10.1097/QAD.0b013e328353b066.

\title{
Lopinavir/ritonavir Monotherapy After Virologic Failure of First- line Antiretroviral Therapy in Resource-limited Settings
}

\author{
John A. Bartlett ${ }^{a, b}$, Heather J. Ribaudoc, Carole L. Wallis ${ }^{d}$, Evgenia Agac, David K. \\ Katzenstein $^{\mathrm{e}}$, Wendy S. Stevens ${ }^{\dagger}$, Michael R. Norton ${ }^{\mathrm{g}}$, Karin L. Klingman ${ }^{\mathrm{h}}$, Mina C. \\ Hosseinipouri, John A. Crump ${ }^{a, b}$, Khuanchai Supparatpinyoj, Sharlaa Badal-Faesen ${ }^{f}$, \\ Beatrice A. Kallungal ${ }^{k}$, and Nagalingeswaran Kumarasamy
}

aDuke University Medical Center bKilimanjaro Christian Medical Centre 'Statistical Data Analysis Center, Harvard University d Lancet Laboratories, South Africa eStanford University fUniversity of Witswatersrand ${ }^{g}$ Abbott hDivision of AIDS, National Institutes of Health 'University of North Carolina- Chapel Hill and UNC Project, Lilongwe, Malawi ${ }^{j}$ Research Institute for Health Sciences, Chiang Mai University kSocial and Scientific Systems 'YRG Centre for AIDS Research and Education.

\section{Abstract}

Objective-To evaluate virologic response rates of lopinavir/ritonavir (LPV/r) monotherapy as second-line antiretroviral treatment (ART) among adults in resource-limited settings (RLS).

Design-Open-label pilot study of LPV/r monotherapy in participants on first-line nonnucleoside reverse transcriptase inhibitor 3-drug combination ART with plasma HIV-1 RNA 1000-200 000 copies/mL.

Methods-Participants were recruited from 5 sites in Africa and Asia within the AIDS Clinical Trials Group (ACTG) network. All participants received LPV/r 400/100mg twice daily. The primary endpoint was remaining on LPV/r monotherapy without virologic failure (VF) at week 24. Participants with virologic failure were offered addition of emtricitabine and tenofovir (FTC/ TDF) to LPV/r.

(C) 2012 Wolters Kluwer Health | Lippincott Williams \& Wilkins

Correspondence to John A. Bartlett, Box 3238, Duke University Medical Center, Durham, NC 27710 USA. Tel: +919 681 8043; fax: +1919681 7748; jab5@duke.edu.

ICJME criteria for authorship:

1) Substantial contributions to the conception and design, acquisition of data, or analysis and interpretation of data.

2) Drafting the article or revising it critically for important intellectual content.

3) Final approval of the version to be published.

Author contributions:

John A. Bartlett 1,2,3

Evgenia Aga 1,2,3

Heather Ribaudo 1,2,3

Carole Wallis 1,2,3

David K. Katzenstein 1,2,3

Wendy Stevens 1,2,3

Michael Norton 1,2,3

Karin Klingman 1,2,3

Mina Hosseinipour 1,2,3

John Crump 1,2,3

Khuanchi Supparatpinyo 1,2,3

Beatrice Kallungal 1,2,3

Nagalingeswaran Kumarasamy 1,2,3 
Results-Mutations associated with drug resistance were encountered in nearly all individuals screened for the study. One hundred and twenty-three participants were enrolled, and 122 completed 24 weeks on study. A high proportion remained on LPV/r monotherapy without VF at 24 weeks $(87 \%)$. Archived samples with HIV-1 RNA levels $<400$ at week $24(\mathrm{n}=102)$ underwent ultrasensitive assay. Of these subjects, 62 had levels $<40$ copies $/ \mathrm{mL}$ and 30 had levels 40-200. Fifteen subjects experienced VF, among whom 11 had resistance assessed, and 2 had emergent protease inhibitor mutations. The presence of baseline thymidine analogue mutations and K65R predicted a lower VF rate. Thirteen subjects with VF added FTC/TDF, and 1 subject added FTC/ TDF without VF. At study week 48, 11/14 adding FTC/TDF had HIV-1 RNA levels < 400 copies $/ \mathrm{mL}$.

Conclusions-In this pilot study conducted in diverse RLS, LPV/r monotherapy as second-line ART demonstrated promising activity.

\section{Introduction}

More than five million HIV-1-infected persons are currently receiving antiretroviral therapy (ART) globally [1], resulting in dramatic reductions of HIV-1-related morbidity and mortality. The World Health Organization (WHO) recommends a simplified public health approach to ART prescription to reach HIV-1-infected persons who live in resource-limited settings (RLS) [2]. First-line ART is suggested with a non-nucleoside reverse transcriptase inhibitor (NNRTI) and two nucleoside/ nucleotide reverse transcriptase inhibitors (NRTIs), representing potent, safe and relatively inexpensive regimens. Virologic suppression rates on first-line ART commonly exceed $80 \%$ at one year on treatment among patients who are retained in care [3]. In the event of first-line ART failure, second-line ART is available in many RLS, which consists of a boosted protease inhibitor (PI)- containing regimen in combination with two NRTIs. Second-line ART also provides high rates of plasma HIV- 1 RNA suppression at 1 year, with approximately $75 \%$ of patients achieving levels $<400$ copies/mL if they remain in care [3].

Despite the therapeutic successes of ART, considerable challenges remain. Monitoring of plasma HIV-1 RNA levels is not available in most RLS, and as a result, first-line ART failure is often identified by either immunological or clinical changes that may occur long after loss of virologic suppression. The delay in identifying ART failure has been shown to lead to the accumulation of complex resistance profiles that impact susceptibility to NRTIs and their future use [4-8]. It is unknown to what extent the inclusion of NRTI in second-line ART regimens contributes to antiretroviral activity given these observations. In the worstcase scenario, NRTIs may only expose patients to the increased risk of drug-related toxicities and add cost to second-line ART.

Previous studies of boosted PI monotherapy in treatment naïve HIV-1-infected persons have suggested that most adherent patients can achieve significant virologic suppression, albeit less robust than that achieved with 3 drug, multi-class drug combinations [9,10]. Interestingly, despite observations of low-level viremia among boosted PI monotherapy recipients, studies to date have rarely identified the evolution of drug resistance mutations in the HIV protease gene [11]. In RLS with limited availability of alternative antiretroviral medications and extensive NRTI resistance following first-line virological failure, boosted PI monotherapy could represent a viable option for second-line ART. The A5230 study of the AIDS Clinical Trial Group (ACTG) was designed to evaluate the activity and tolerability of second-line ART with lopinavir/ritonavir (LPV/r) monotherapy in a pilot study among persons experiencing virologic failure on a first-line regimen containing an NNRTI and two NRTIs. The study has a 24 week primary endpoint with continued long-term follow-up to 2 years; here we present the primary analysis results. 


\section{Methods}

\section{Study design}

ACTG A5230 is an open-label, multicenter pilot study of LPV/r monotherapy in subjects failing a first-line regimen containing an NNRTI and two NRTIs, (NCT00357552). The study was conducted at five Clinical Research Sites, including three sites in Africa (Lilongwe, Malawi; Moshi, Tanzania; and Johannesburg, South Africa) and two sites in Asia (Chiang Mai, Thailand; and Chennai, India). The primary objective of the study was to demonstrate that LPV/r monotherapy provides at least a $65 \%$ virologic response rate in this patient population, defined as achieving and maintaining HIV-1 RNA levels $<400$ copies/ $\mathrm{mL}$ for at least 24 weeks while on LPV/r monotherapy.

\section{Eligible subjects}

Eligible subjects had documented HIV-1 infection with 2 methods of antibody and/or antigen or nucleic acid detection, and were 18 years or older. They must have received a first-line regimen containing an NNRTI and two NRTIs for at least 6 months continuously, and have a plasma HIV RNA-1 level between 1000 and 200000 copies $/ \mathrm{mL}$ within 30 days of study entry. Eligible subjects were also required to have the following laboratory values; hemoglobin $>8 \mathrm{mg} / \mathrm{dL}$, platelets $>50000 / \mathrm{mm}^{3}$, estimated creatinine clearance $>60 \mathrm{~mL} /$ minute, AST, ALT and alkaline phosphatase $<3$ times the upper limit of normal, and total bilirubin $<2.5$ times the upper limit of normal. Pregnant or breastfeeding women, and persons with prior PI use, a serious medical condition within the previous 14 days, active substance abuse, or evidence of chronic hepatitis B infection as documented by a positive hepatitis B surface antigen result were excluded. All subjects provided written informed consent.

\section{Study evaluations}

For the first 24 weeks following LPV/r treatment initiation, subjects returned at weeks 2 and 4 , and then every 4 weeks until week 24 . At every study visit a clinical assessment, list of concomitant medications, adherence interview using the ACTG Adherence Questionnaire [12] and pill count were performed. Serum chemistries, liver enzymes and hematology values were measured at weeks 4, 8, 12 and 24 . Fasting lipid panels were done at study entry, and weeks 12 and 24. Plasma HIV-1 RNA levels were measured at study entry, and weeks 12, 16, 20 and 24. CD4+ cell counts were measured at study entry, and weeks 16 and 24. All laboratories participated in international quality assurance testing. With the exception of samples drawn at week 2 and 4, quantitation of HIV-1 RNA levels in A5230 was performed on-site in real time using a US Food and Drug Administration- approved HIV-1 RNA assay; week 2 and 4 samples were tested at the time of the week 12 sample. In a post-hoc analysis, all week 24 samples with plasma HIV RNA-1 $<400$ copies $/ \mathrm{mL}$ underwent further evaluation with the Abbott m2000rt RealTime ${ }^{\mathrm{TM}}$ assay, (Abbott Laboratories, Abbott Park, Illinois USA; lower limit of detection 40 copies $/ \mathrm{mL}$ ) in one of three designated laboratories. Samples for genotypic resistance testing were collected on all subjects at the screening visit. Resistance testing was performed in-batch at two regional laboratories using ViroSeq (Celera Diagnostics, CA) as per manufacturer's instructions. Briefly, a $1.7 \mathrm{~kb}$ amplicon was generated by a reverse transcriptase (RT)-initiated polymerase chain reaction encompassing the entire protease (PR) and partial reverse transcriptase (RT). Sequencing was performed with an ABI Prism 3100-Avant Genetic Analyzer (Applied Biosystems, USA). HIV-1 subtype and drug resistance were determined from PR and RT sequences, and analyzed using the Stanford Algorithm [13,14]. 


\section{Study interventions}

All subjects received fixed dose combination (FDC) LPV/r $400 \mathrm{mg} / 100 \mathrm{mg}$ twice daily. If subjects developed virologic failure, FDC emtricitabine $200 \mathrm{mg} / \mathrm{tenofovir} 300 \mathrm{mg}$ (FTC/ TDF) daily was added to LPV/r. Concomitant treatment with rifampin and other medications listed in the LPV/r package insert was prohibited.

\section{Primary endpoint}

The primary endpoint was remaining on LPV/r monotherapy without virologic failure at week 24. Virologic failure was defined as 1) failure to suppress plasma HIV-1 RNA to $<400$ copies/mL by week 24 , or 2) confirmed rebound of plasma HIV-1 RNA levels to $>400$ copies $/ \mathrm{mL}$ after confirmed suppression to $<400$ copies $/ \mathrm{mL}$. With some exceptions per protocol, discontinuation of LPV/r monotherapy was considered as a primary endpoint; exceptions included discontinuation for rifampin-based tuberculosis treatment, addition of antiretroviral drugs due to pregnancy, and death with HIV-1 RNA levels $<400$ copies $/ \mathrm{mL}$.

\section{Sample size}

The target sample size of 120 subjects was chosen to provide at least $90 \%$ power to show that LPV/r monotherapy could provide at least a $65 \%$ success rate over 24 weeks. This assumed that the true success rate of the strategy in this population is $76 \%$, and used a onesided type I error rate of 5\%.

\section{Statistical analysis}

Baseline characteristics of the study population were compared across sites using nonparametric $k$-sample tests. The proportion of subjects remaining on LPV/r monotherapy without virologic failure at week 24 was estimated with an exact $95 \%$ confidence interval. The proportion of subjects with plasma HIV-1 RNA levels $<400$ copies/mL over time was estimated using intent to treat methods with missing/off treatment evaluation ignored ( $\mathrm{M}=$ I) and considered as failure ( $>400$ copies/ $\mathrm{ml})(\mathrm{M}=\mathrm{F})$; as treated analyses were also performed. Exact logistic regression was used to assess factors associated with the probability of failure; prior use of efavirenz (EFV) versus NVP, prior therapy duration (> 3 years, $1-3$ years and $<1$ year), sex, baseline CD4+ cell count ( $<200$ vs. $>200 / \mathrm{mm}^{3}$ ), baseline plasma HIV-1 RNA levels (<10 000 vs. $>10000$ copies $/ \mathrm{mL}$ ), and NRTI resistance (defined as K65R or thymidine analogue mutations (TAMs) vs. no NRTI resistance). In unplanned post-hoc analyses, baseline HIV-1 RNA levels and rates of failure are described. All analyses were restricted to data from study entry to the scheduled week 24 visit. In order to provide information on the consequences of LPV/r monotherapy failure, the HIV- 1 RNA and CD4+ cell count responses, and resistance findings through week 48 are included from subjects reaching a primary failure endpoint.

\section{Results}

\section{Baseline characteristics}

One hundred twenty-three subjects entered the study; their baseline characteristics are described in Table 1. The majority of subjects were female. The most common first-line ART regimen was nevirapine, lamivudine (3TC) and stavudine (d4T)-containing regimens $(\mathrm{n}=63,51 \%)$, and the majority of subjects had received ART for $>3$ years $(\mathrm{n}=70,57 \%)$. The median plasma HIV-1 RNA level was $4.34 \log _{10}$ copies $/ \mathrm{mL}$, and 21 subjects (17\%) had levels $>100000$ copies $/ \mathrm{mL}$. The median CD4+ cell count at study entry was $164 / \mathrm{mm}^{3}$, and 70 subjects $(57 \%)$ had counts $<200 / \mathrm{mm}^{3}$. Screening plasma HIV-1 RNA levels, the proportion of subjects with baseline CD4+cell counts below $100 / \mathrm{mm}^{3}$, and the proportion of subjects meeting WHO CD4+ cell count criteria for regimen failure [15] were found to 
differ by site $(\mathrm{P}=0.016, \mathrm{P}<0.001, \mathrm{P}=0.002, \mathrm{P}<0.001$, respectively), with subjects from the South African site having significantly lower values for each parameter.

\section{Baseline resistance and subtyping}

From the 114 screening samples that were successfully sequenced, nearly all subjects had mutations associated with drug resistance. Most subjects were found to have mutations associated with reduced susceptibility to 3TC $(n=108 ; 95 \%)$, EFV $(n=112 ; 98 \%)$ and NVP $(n=112 ; 98 \%)$. Based on genotyping of the PR and partial RT, HIV-1 subtype $C$ was found to be the most prevalent (66\%), followed by AE (19\%), A1 (7\%) and D (6\%).

\section{Subject disposition}

Of 123 subjects who entered the study, 122 completed 24 weeks of study follow-up; one subject died from a myocardial infarction deemed unrelated to treatment with a plasma HIV-1 RNA level $<400$ copies/mL at week 20 . All subjects remained on study treatment (LPV/r-monotherapy or LPV/r with FTC/TDF) throughout the 24-week primary endpoint.

\section{Virologic responses}

At week 24, 107 (87\%) subjects remained on LPV/r monotherapy without virologic failure (95\% CI: 80- 92\%). From week 16, >80\% of subjects had plasma HIV- 1 RNA levels < 400 copies $/ \mathrm{mL}$ regardless of how missing data and premature treatment discontinuations were analyzed (Fig. 1). Of note, 95/102 subjects (93\%) entering with baseline plasma HIV-1 RNA levels $<100000$ copies/mL had achieved HIV-1 RNA $<400$ copies/mL by week 16 compared to 19/21 (90\%) with baseline levels $>100000$ copies $/ \mathrm{mL}$. One hundred and two subjects had plasma HIV-1 RNA levels $<400$ copies/mL at week 24 . Among these 102 subjects (panel within Fig. 1), 62 (61\%) had plasma HIV-1 RNA <40 copies/mL, 30 (29\%) had levels 40-200 copies $/ \mathrm{mL}, 7$ (7\%) had levels 201-400 copies/mL, and $3(3 \%)$ had levels $>400$ copies/mL, (respectively 413, 489 and 1712 copies $/ \mathrm{mL}$ ).

\section{Virologic failures}

Fifteen subjects met the criteria for virologic failure at week 24, and an additional subject had FTC/TDF added without virologic failure (total failures 16, 13\%), (Fig. 2). Failure occurred in 6/21 subjects (29\%) entering with baseline plasma HIV-1 RNA levels $>100000$ copies/mL; in contrast, in the 102 subjects with baseline plasma HIV-1 RNA levels $<100$ 000 copies/ml, 10 subjects (10\%) met virologic failure criteria. Among the 15 subjects with virologic failure, PR and RT sequences were obtained from 11 (73\%); one individual had insufficient sample available, and 3 samples could not be sequenced [HIV-1 RNA level $<1000$ (2 subjects) and 1330 copies/mL (1 subject)]. PR mutations not observed at study entry were identified in two subjects; V82F in one and L33F, M46I, I54V, V82A and L90M in the second, (Table 2). With regard to NRTI-associated mutations at the time of virologic failure, eight subjects had between 1 and 6 mutations that were no longer detectable compared to mutations detected at screening. Five subjects gained one NRTI-associated mutation from study entry to virologic failure. From study entry to virologic failure, no NNRTI-associated mutations were gained by any of the subjects and in six subjects NNRTI mutations were no longer detectable.

\section{Predictors of virologic failure}

In unadjusted and adjusted analyses, no associations between the potential predictors and virologic failure were apparent $(\mathrm{P}>0.10)$. 


\section{Treatment intensification}

By study week $48,14(88 \%)$ of the 16 primary endpoint failures intensified their treatment with FTC/TDF and 11 (79\%) of 14 suppressed to $<400$ copies/mL following intensification (Fig. 2). Among the three subjects who failed to suppress, one (33\%) had newly emergent PI-associated mutations at the time of virologic failure. Of the subjects who suppressed to $<400$ copies $/ \mathrm{mL}$ on treatment intensification, all were suppressed at study week 48 . Table 2 lists the resistance profiles for subjects at screening and at virologic failure, categorized by receipt of treatment intensification and virologic response. Among the 11 subjects who suppressed HIV-1 RNA to <400 copies/mL, seven had at least one major NRTI-associated resistance mutation that could no longer be detected by population-based sequencing. Among the two subjects who did not suppress HIV-1 RNA levels to $<400$ copies $/ \mathrm{mL}$, both either maintained the same number of NRTI-associated resistance mutations or gained a new mutation.

\section{CD4+ cell count responses}

CD4+ cell counts increased from a median (Q1-Q3) of $164(82-268) / \mathrm{mm}^{3}$ at study entry to a median of $268(169-404) / \mathrm{mm}^{3}$ at week 24 . Among subjects remaining on LPV/rmonotherapy without primary endpoint failure at week 24 , the median CD4+ cell count increase over 24 weeks was 102 cells $/ \mathrm{mm}^{3}$ (46-157) compared to 111 (35-172) for the primary endpoint failures.

\section{Adherence}

Adherence levels were generally high throughout the 24 weeks; by self-report, $79-90 \%$ of subjects responded that they had never missed a dose of study medication at each adherence assessment. Hypothesis-testing statistical analyses were not performed but there appeared to be no differences in reported adherence between subjects who were virologic successes versus failures.

\section{Safety and tolerability}

Thirty-one subjects (25\%) experienced grade 3 or 4 adverse events during LPV/r monotherapy (Table 3 ). The most common events included lipid elevations (1 subject with grade 3 fasting triglycerides, 6 subjects with grade 4 fasting triglycerides, 5 subjects with grade 3 fasting LDL cholesterol, and 5 subjects with grade 3 fasting total cholesterol), elevations in fasting blood sugar ( 2 subjects with grade 3 and 4 subjects with grade 4 ), and abnormalities in serum phosphorus ( 5 subjects with grade 3 hypophosphatemia).

\section{Discussion}

This pilot study, conducted at 5 geographic sites with multiple HIV-1 subtypes represented, demonstrates promising antiretroviral activity of LPV/r monotherapy as second-line ART in NNRTI-experienced HIV-infected persons. Over 24 weeks, $87 \%$ of subjects remained on LPV/r monotherapy with plasma HIV-1 RNA levels suppressed to $<400$ copies $/ \mathrm{mL}$ with the lower limit on the estimated efficacy of the regimen of $80 \%$, exceeding our target threshold of $65 \%$. Among subjects who developed virologic failure, treatment intensification with FDC FTC/TDF resulted in suppression to $<400$ copies/mL over 48 weeks for the majority of subjects.

As the number of HIV-1-infected persons on first-line ART grows, inevitably some will fail and need second line ART. Currently the numbers of patients switching to second-line regimens are relatively low, estimated at 3-5\% [16-18], although it is likely that $15-25 \%$ of HIV-infected persons on first-line ART for at least 12 months have detectable plasma HIV-1 RNA [18]. When plasma HIV-1 RNA monitoring is available, it does lead to earlier 
switches to second-line ART [18], and better outcomes have been associated with RNA $<1000$ copies/mL at the time of switching [19]. Unfortunately, in many RLS settings RNA measurement is not available and first-line ART failure is recognized late, with the consequence of increasing patient numbers with extensive NRTI and NNRTI resistance mutations.

Other studies of second-line ART in RLS have demonstrated similar rates of virologic suppression with use of LPV/r plus NRTI [19-21]. One study examined outcomes in 328 subjects receiving LPV/r, zidovudine and didanosine as second-line ART; plasma HIV-1 RNA levels were available for 262 subjects, and 203 (77\%) had levels $<400$ copies $/ \mathrm{mL}$ after 1 year, comparable to our results with LPV/r monotherapy [19]. Another study of secondline ART in 141 South African subjects included the use of LPV/r and genotypic resistance testing to guide the choice of the two NRTI's [20]. Baseline characteristics of the South African study population were similar to ACTG A5230 subjects with a median plasma HIV-1 RNA of 17000 copies/mL and 17\% with RNA >100 000 copies/mL, although NRTI and NNRTI resistance was not as extensive as observed in our population. Seventy-seven percent of these subjects suppressed to $<400$ copies $/ \mathrm{mL}$ and $65 \%$ to $<50$ copies $/ \mathrm{mL}$ at week 24 , similar to the $87 \%$ of subjects with $<400$ copies $/ \mathrm{mL}$ and $50 \%(62 / 123)$ with $<40$ copies/ $\mathrm{mL}$ observed in ACTG 5230. A third study of second-line ART with LPV/r, zidovudine, lamivudine and TDF among 109 persons in Malawi found that $85 \%$ of those surviving at one year had plasma HIV-1 RNA levels $<400$ copies/mL [21].

Previous trials of LPV/r monotherapy have observed findings of low-level viremia in firstline regimens, and randomized trials have demonstrated such viremia is significantly greater with monotherapy compared to 3- drug LPV/r-containing regimens [9,10]. When week 24 samples for subjects remaining on LPV/r monotherapy with plasma HIV-1 RNA levels $<400$ copies $/ \mathrm{mL}$ in A5230 were re-tested using an ultrasensitive assay, low-level viremia was detected in 39\% with the majority of samples measured in the $40-200$ copies $/ \mathrm{mL}$ range. The significance of low-level viremia is uncertain, with other trials of LPV/r maintenance monotherapy suggesting that it may remain stable for prolonged periods of time [22,23]. Numerous studies have demonstrated that resistance mutations in the protease gene are infrequent in persons receiving boosted protease inhibitors [24], even as monotherapy, despite this low-level viremia [11,23]. Similar observations were made in ACTG A5230only $7 \%(2 / 16)$ subjects virologically failing LPV/r monotherapy had protease mutations that were not observed at study entry.

A recent Thai study randomized subjects failing first-line ART to either LPV/r monotherapy or LPV/r, FTC and TDF, and was conducted simultaneously with ACTG A5230 [25]. They observed that the proportion of subjects with viremia at levels 50-400 after 48 weeks was significantly higher in the monotherapy arm (14\% versus $3 \%)$, leading them to conclude that 3-drug second-line regimens were superior. A second randomized trial of LPV/r maintenance monotherapy versus combination therapy (SARA trial) was conducted in Uganda and Zimbabwe among 192 subjects who had received 24 weeks of second-line combination therapy including LPV/r [26]. Plasma HIV-1 RNA was not monitored in realtime, but testing of archived specimens revealed that the combination therapy group had greater virologic suppression at week 24 (77\% vs. $60 \%<50$ copies $/ \mathrm{mL})$. Genotypic resistance testing on 10 monotherapy subjects with plasma HIV-1 RNA levels $>1000$ copies/ $\mathrm{mL}$ revealed that a minority of subjects had new protease mutations at the time of failure. Treatment intensification was not included in the SARA trial design, and the availability of real-time plasma HIV-1 RNA monitoring and intensification represent an important distinguishing feature of ACTG A5230. 
The short-term virologic responses to FTC/TDF intensification in ACTG A5230 subjects are encouraging, and similar responses were observed in the Thai trial where 8/17 subjects in the LPV/r monotherapy arm suppressed to $<50$ copies $/ \mathrm{mL}$ following intensification with FTC/TDF [25] and in trials of LPV/r monotherapy when NRTIs were added in subjects with low-level viremia $[23,24]$. The strategy LPV/r monotherapy followed by treatment intensification in the event of virologic failure may be critically important for future studies of LPV/r monotherapy. However, this strategy does impose the need for plasma HIV-1 RNA monitoring and associated costs that must be balanced against the high costs and potential toxicities of second-line regimens which empirically include NRTIs [27]. An intriguing association between reversion of NRTI resistance mutations detectable by population sequencing at virologic failure and response to FTC/TDF intensification in ACTG A5230, perhaps suggesting that some degree of re-sensitization may occur, although longer periods of observation are needed to assess the durability of responses and the potential impact of minor variants on longer-term outcomes. The continued follow-up of all subjects over 2 years on ACTG A5230 will provide more information on the durability of the strategy of $\mathrm{LPV} / \mathrm{r}$ monotherapy with intensification with FTC/TDF.

This study does have a number of limitations. It is a pilot, open-label uncontrolled study, and the results must be cautiously interpreted. A larger randomized trial comparing secondline ART with LPV/r monotherapy followed by FTC/TDF intensification for inadequate responses versus 3-drug LPV/r-containing ART is warranted. Further, the more intensive monitoring and support provided in a clinical trial may limit generalizing to responses in clinical cohorts. Finally, at the present time we are only able to report results through the primary endpoint follow-up to week 24; follow-up in ACTG A5230 is ongoing and will continue until each subject has reached 104 weeks (expected in April 2012).

In summary, persons failing first-line ART with NNRTI-containing regimens have extensive NRTI and NNRTI resistance mutations, which may carry important implications for secondline ART choices. These pilot results suggest promising early responses to the strategy of second-line LPV/r monotherapy, coupled with further virologic suppression following intensification with FTC/TDF among persons with virologic failure.

\title{
Acknowledgments
}

\author{
Conflicts of interest
}

The project described was funded by award U01AI068636 from the National Institute for Allergy and Infectious Diseases, and was further supported by the National Institute of Mental Health and the National Institute of Dental and Craniofacial Research. The content is solely the responsibility of the authors and does not necessarily represent the official views of the National Institute of Allergy and Infectious Diseases or the National Institutes of Health. The authors wish to thank all ACTG A5230 study subjects for their participation. In addition, we thank the Clinical Research Site members and their supporting grants at the 5 sites who conducted ACTG A5230 (Drs. Agnes Moses and Albert Mwafongo, UNC Project Lilongwe, U01AI069518; Drs. Venance Maro and John Shao, KCMC, U01069484; Professor Thira Sirisanthana and Ms. Patchanee Samutarlai, Chiang Mai University; Ms. Petronilla Borain and Vuyokazi Jezile, University of Witswatersrand, U01AI38858; Ms. Jabin Sharma and Dr. Poongulali, YRG Care, U01AI069432) and additional members of the ACTG A5230 Protocol Team (Heather Vezina, Amy Kambrink, Bernadette Jarocki, Vicki Bailey, A.K. Shrikrishnan, and Eric Lawrence). JAB is supported by NIH awards U01 AI069484, P30 AI64518, D43 TW006732, D43 CA153722, and HRSA award T84HA21123. Michael Norton is an employee of Abbott.

Funding support received from the National Institute for Allergy and Infectious Diseases of the United States National Institutes of Health, and Abbott. Drug supplies were provided by Abbott and Gilead Sciences, Inc.

\section{References}

1. [accessed on November 25, 2011] http://www.globalhealthfacts.org/data/topic/ map.aspx $?$ ind $=10$

2. [accessed on November 25, 2011] http://whqlibdoc.who.int 
3. Bartlett J, Shao J. Success, challenges and limitations of antiretroviral therapy in resource-limited settings. The Lancet Infectious Diseases. 2009; 9:637-649. [PubMed: 19778766]

4. Sungkanuparph S, Manosuthi W, Kiertiburanakul S, Piyavong B, Chumpathat N, Chantratita W. Options for second-line antiretroviral regimens for HIV type-1 infected patients whose initial regimen of a fixed-dose combination of stavudine, lamivudine and nevirapine fails. Clin Inf Dis. 2007; 44:447-452.

5. Marconi V, Sunpath H, Li Z, et al. Prevalence of HIV-1 drug resistance after failure of a first highly active antiretroviral therapy regimen in KwaZulu Natal, South Africa. Clin Inf Dis. 2008; 46:15891597.

6. Kumarasamy N, Madhavan V, Venkatesh K, et al. High frequency of clinically significant mutations after first-line generic highly active antiretroviral therapy failure: Implications for second-line options in resource-limited settings. Clin Inf Dis. 2009; 49:306-309.

7. Hosseinipour M, van Oosterhout J, Weigel R, et al. The public health approach to identify antiretroviral therapy failure: High level nucleoside reverse transcriptase resistance among Malawians failing first-line antiretroviral therapy. AIDS. 2009; 23:1127-1134. [PubMed: 19417582]

8. Wallis CL, Mellors JW, Venter F, Sanne I, Stevens W. Varied HIV Drug resistance patterns among patients on failing antiretroviral therapy in the South African national roll-out programme. J Acquir Immune Defic Syndr. 2010; 53:480-484. [PubMed: 19801944]

9. Delfraissy J, Flandre P, Deluagerre C, et al. Lopinavir/ritonavir monotherapy or plus zidovudine and lamivudine in antiretroviral-naïve HIV-infected patients. AIDS. 2008; 22:385-393. [PubMed: 18195565]

10. Ghosn J, Flandre P, Cohen-Codar I, et al. Long-term (96 week) follow-up of antiretroviral-naïve HIV-infected patients treated with first-line lopinavir/ritonavir monotherapy in the MONARK trial. HIV Med. 2010; 11:137-142. [PubMed: 19682100]

11. Deluagerre C, Flandre P, Chaix M, et al. Protease inhibitor resistance analysis in the MONARK trial comparing first-line lopinavir-ritonavir monotherapy to lopinavir-ritonavir plus zidovudine and lamivudine triple therapy. Antimicrob Agents Chemother. 2009; 53:2934-2939. [PubMed: 19451297]

12. Chesney M, Ickovics J, Chambers D, et al. Self-reported adherence to antiretroviral medications among participants in HIV clinical trials: the AACTG Adherence Instruments. AIDS CarePsychological and Socio-Medical Aspects of HIV/AIDS. 2000; 12:255-266.

13. Rhee S-Y, Gonzales M, Kantor R, Betts B, Ravela J, Shafer R. Human immunodeficiency virus reverse transcriptase and protease sequence database. Nucleic Acids Research. 2003; 31:298-303. [PubMed: 12520007]

14. Shafer R. Rationale and Uses of a Public HIV Drug-Resistance Database. Journal of Infectious Diseases. 2006; 194:S51-S58. [PubMed: 16921473]

15. [accessed November 25, 2011] WHO Consultation on ART Failure in the Context of the Public Health Approach. at www.who.int

16. Pujades-Rodriguez M, O’Brien D, Humblet P, Calmy A. Second-line antiretroviral therapy in resource-limited settings: The experience of Medecins Sans Frontieres. AIDS. 2008; 22:13051312. [PubMed: 18580610]

17. Harries A, Zachariah R, van Oosterhout J, et al. Diagnosis and management of antiretroviraltherapy failure in resource-limited settings in sub-Saharan Africa: Challenges and perspectives. The Lancet Inf Dis. 2010; 10:60-65.

18. Keiser O, Orrell C, Egger M, et al. Public-health and individual level approaches to antiretroviral therapy: township South Africa and Switzerland compared. PLoS Med. 2008; 5:e148. [PubMed: 18613745]

19. Fox M, Ive P, Long L, Maskew M, Sanne I. High rates of survival, immune reconstitution and virologic suppression on secondline antiretroviral therapy in South Africa. J Acquir Immun Def Syndr. 2010; 53:500-506.

20. Murphy R, Sunpath H, Lu Z, et al. Outcomes after virologic failure of first-line ART in South Africa. AIDS. 2010; 24:1007-1012. [PubMed: 20397305] 
21. Hosseinipour M, Kumwenda J, Weigel R, et al. Second-line treatment in the Malawi antiretroviral programme: High early morality, but good outcomes in survivors, despite extensive drug resistance at baseline. HIV Med. 2010; 11:510-518. [PubMed: 20345885]

22. Pulido F, Arribas J, Delgado R, et al. Lopinavir-ritonavir montherapy versus lopinavir-ritonavir and two nucleosides for maintenance therapy of HIV. AIDS. 2008; 22:1-9. [PubMed: 18090386]

23. Arribas J, Delgado R, Arranz A, et al. Lopinavir-ritonavir monotherapy versus lopinavir-ritonavir and 2 nucleosides for maintenance therapy of HIV: 96-week analysis. J Acquir Immune Defic Syndr. 2009; 51:147-152. [PubMed: 19349870]

24. Wallis CL, Mellors JW, Venter WD, Sanne I, Stevens W. Protease inhibitor resistance is uncommon in HIV-1 subtype C infected patients on failing second-line lopinavir/r-containing antiretroviral therapy in South Africa. AIDS Res Treat. 2011; (2011):769627. [PubMed: 21490784]

25. Bunupuradah, T.; Chetchotisakd, P.; Ananworanich, J., et al. Second line lopinavir/ritonavir monotherapy was inferior to tenofovir/ lamivudine//lopinavir/ritonavir in patients who failed NNRTI-regimen: HIV STAR study. Abstract \#584 presented at the Conference on Retroviruses and Opportunistic Infections; February 2011; Boston, MA.

26. Pillay D, Goodall R, Gilks C, et al. Virologic findings from the SARA trial: Boosted PI monotherapy as maintenance second-line ART in Africa. Journ Int AIDS Soc. 2010; 13:O20.

27. Long L, Fox M, Sanne I, Rosen S. The high cost of second-line antiretroviral therapy for HIV/ AIDS in South Africa. AIDS. 2010; 24:915-919. [PubMed: 20042849] 

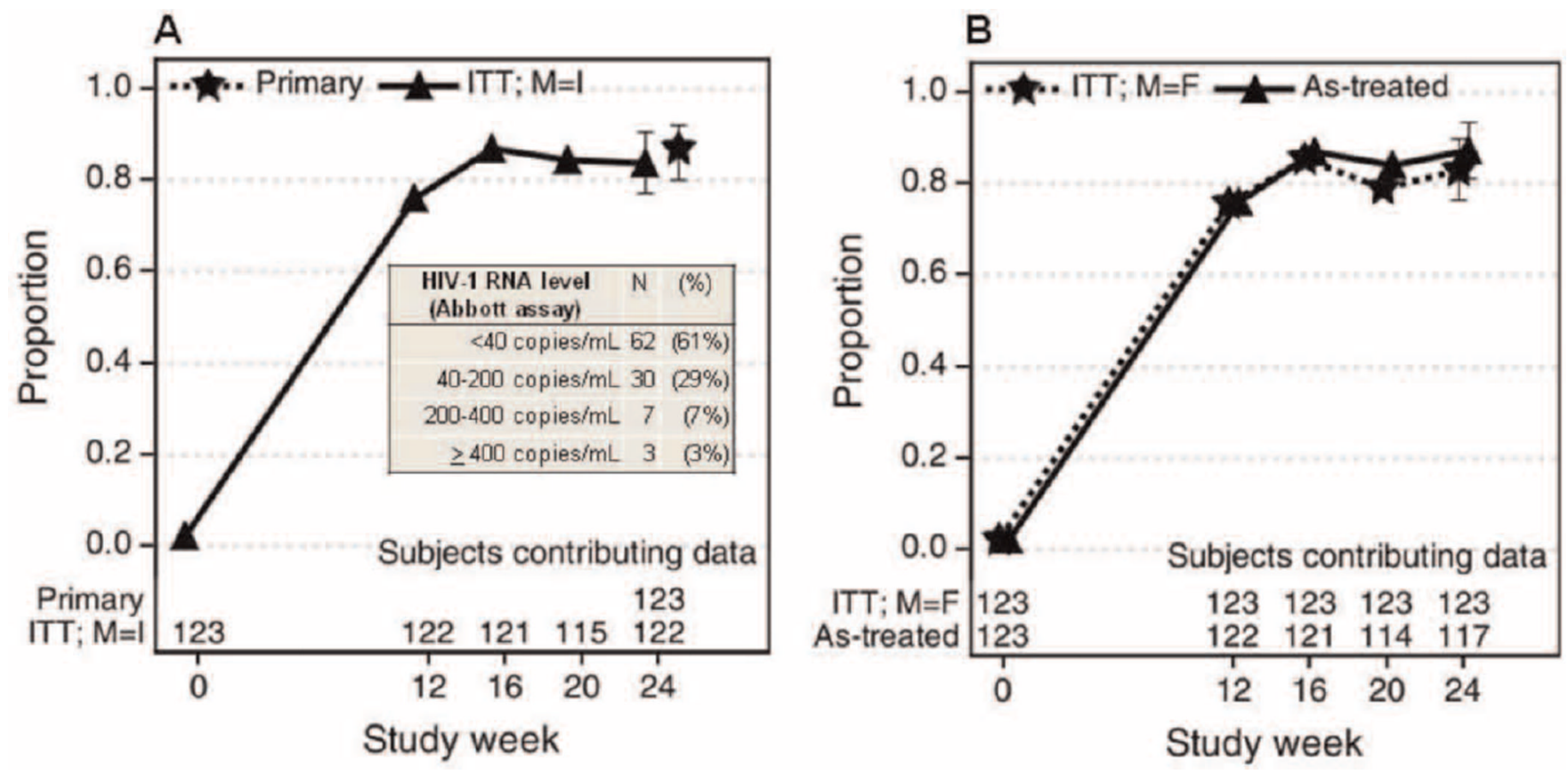

Fig. 1.

Proportion of subjects with HIV-1 RNA levels <400 copies/ml. 




Fig. 2.

Primary endpoint failures. 


\section{Table 1}

Baseline characteristics.

\begin{tabular}{|c|c|c|}
\hline & & Total $(N=123)$ \\
\hline Age (yrs) & $\operatorname{Median}(\mathrm{Q} 1, \mathrm{Q} 3)^{a}$ & $39(33,45)$ \\
\hline Sex & Female & $70(57 \%)$ \\
\hline \multirow[t]{6}{*}{ Prior regimen $b$} & $\mathrm{TDF}+3 \mathrm{TC}+\mathrm{NVP}$ & $4(3 \%)$ \\
\hline & $\mathrm{ZDV}+3 \mathrm{TC}+\mathrm{EFV}$ & $8(7 \%)$ \\
\hline & $\mathrm{ZDV}+3 \mathrm{TC}+\mathrm{NVP}$ & $26(21 \%)$ \\
\hline & $\mathrm{d} 4 \mathrm{~T}+3 \mathrm{TC}+\mathrm{EFV}$ & $21(17 \%)$ \\
\hline & $\mathrm{d} 4 \mathrm{~T}+3 \mathrm{TC}+\mathrm{NVP}$ & $63(51 \%)$ \\
\hline & $\mathrm{ddI}+3 \mathrm{TC}+\mathrm{NVP}$ & $1(1 \%)$ \\
\hline \multirow[t]{4}{*}{ Total ARV exposure } & $\operatorname{Median}(\mathrm{Q} 1, \mathrm{Q} 3)^{a}$ & $3(2,4)$ \\
\hline & $<1$ years & $9(7 \%)$ \\
\hline & $1-<3$ years & $44(36 \%)$ \\
\hline & $3+$ years & $70(57 \%)$ \\
\hline HIV-1 RNA $\left(\log _{10}\right.$ & $\operatorname{Median}(\mathrm{Q} 1, \mathrm{Q} 3)^{a}$ & $4.34(3.75,4.92)$ \\
\hline \multirow[t]{5}{*}{$($ copies/mL)) } & $<3.00$ & $4(3 \%)$ \\
\hline & $3.00-3.99$ & $38(31 \%)$ \\
\hline & $4.00-<4.99$ & $60(49 \%)$ \\
\hline & $5.00-<5.29$ & $14(11 \%)$ \\
\hline & 5.3 & $7(6 \%)$ \\
\hline CD4 cell count & $\operatorname{Median}(\mathrm{Q} 1, \mathrm{Q} 3)^{a}$ & $164(82,268)$ \\
\hline \multirow[t]{4}{*}{$\left(\right.$ cells $\left./ \mathrm{mm}^{3}\right)$} & $<50$ & $19(15 \%)$ \\
\hline & 50-199 & $51(41 \%)$ \\
\hline & $200-349$ & $39(32 \%)$ \\
\hline & 350 & $14(11 \%)$ \\
\hline
\end{tabular}

${ }^{a} \mathrm{Q} 1$ and $\mathrm{Q} 3$ represent the $25^{\mathrm{th}}$ and $75^{\mathrm{th}}$ percentiles, respectively.

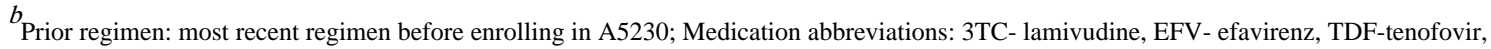
NVP- nevirapine, ZDV- zidovudine, D4T- stavudine, DDI- didanosine. 

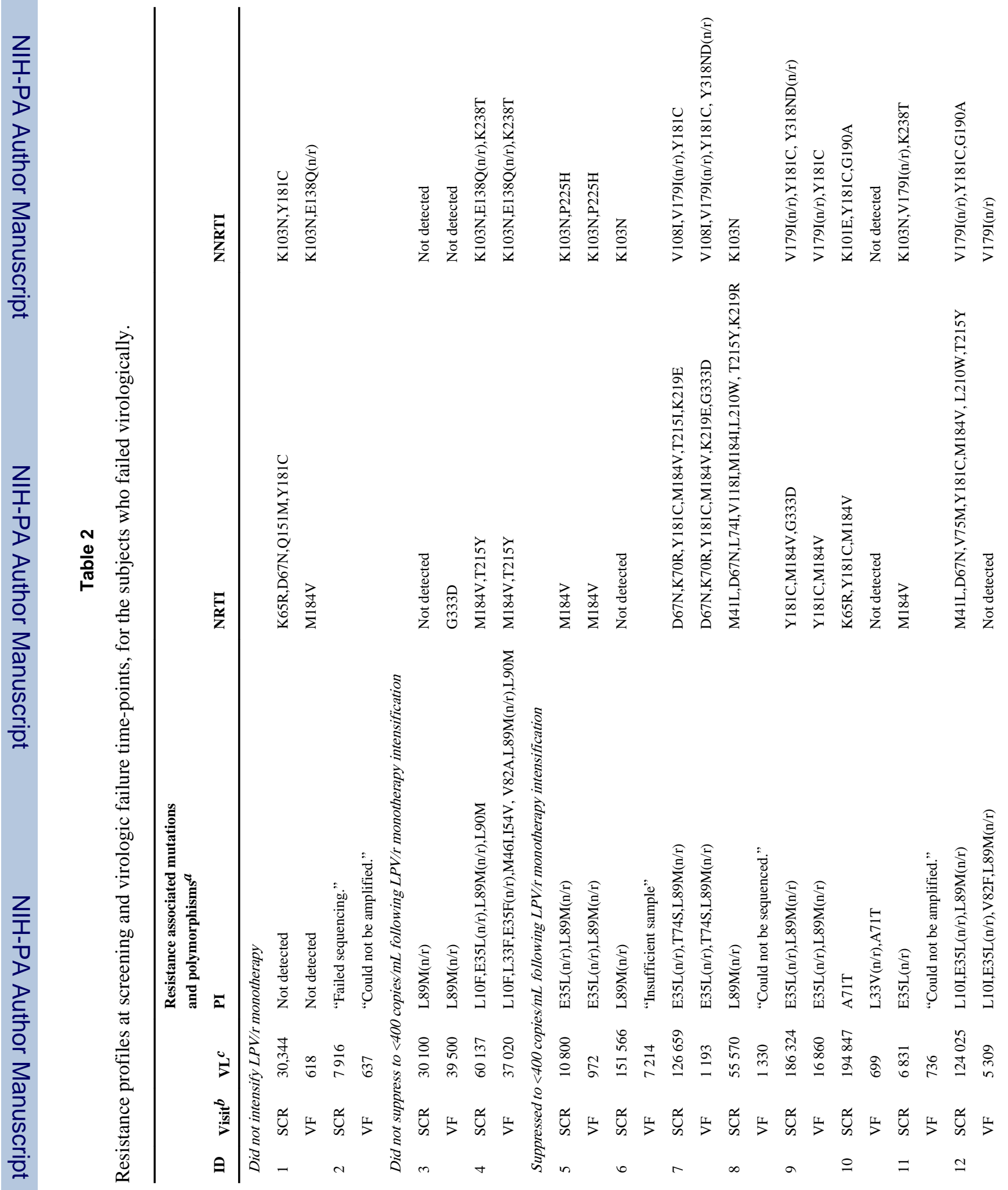


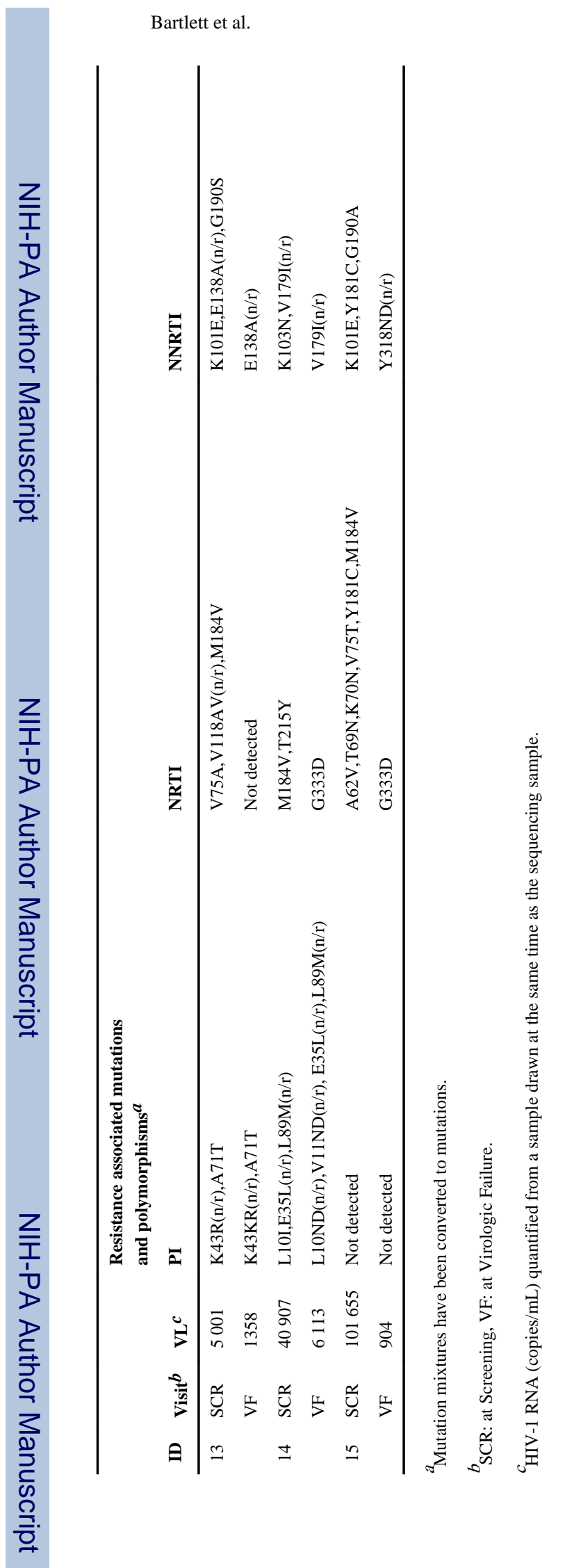

AIDS. Author manuscript; available in PMC 2012 September 17. 


\section{Table 3}

Grade 3 or 4 adverse events during LPV/r monotherapy that were reported for at least $2 \%$ of the study subjects.

\begin{tabular}{|c|c|c|c|}
\hline Sign/Symptom/Laboratory Event & $\begin{array}{l}\text { Number of subjects by toxicity grade } \\
3\end{array}$ & $\begin{array}{l}\text { DAIDS } \\
4\end{array}$ & $\begin{array}{l}\text { Total number }(\%) \\
\text { subjects }\end{array}$ \\
\hline Total Triglycerides (fasting) & 1 & 6 & $7(6 \%)$ \\
\hline Fasting Blood Sugar & 2 & 4 & $6(5 \%)$ \\
\hline LDL (fasting) & 5 & 0 & $5(4 \%)$ \\
\hline Phosphorus & 5 & 0 & $5(4 \%)$ \\
\hline Total Cholesterol (fasting) & 5 & 0 & $5(4 \%)$ \\
\hline SGOT & 1 & 2 & $3(2 \%)$ \\
\hline Absolute Neutrophil Count & 2 & 1 & $3(2 \%)$ \\
\hline Diarrhea/Loose Stools & 2 & 1 & $3(2 \%)$ \\
\hline Total Bilirubin & 2 & 1 & $3(2 \%)$ \\
\hline Hemoglobin & 3 & 0 & $3(2 \%)$ \\
\hline SGPT & 0 & 2 & $2(2 \%)$ \\
\hline Bicarbonate & 1 & 1 & $2(2 \%)$ \\
\hline Potassium & 1 & 1 & $2(2 \%)$ \\
\hline Cachexia/Wasting/weight Loss & 2 & 0 & $2(2 \%)$ \\
\hline
\end{tabular}

Table reflects the number of subjects reporting each category of symptom/lab event; $3=$ Severe, $4=$ Life-Threatening, $5=$ Death. For each subject the worst grade for each event category is reported. 